\title{
Nutri-Seed Priming and Planting Geometry Effects on Growth and Oil Production of Sunflower Varieties under the Ecological Conditions of Tandojam, Sindh, Pakistan
}

\section{Zulfiqar Ali Abbasi ${ }^{*}$, Muhammad Nawaz Kandhro', Aijaz Ahmed Soomro', Naimatullah Leghari², Muhammad Ibrahim Keerio ${ }^{3}$, Ahmed Naqi Shah ${ }^{1}$ and Musrat Begum Abro'}

\section{${ }^{1}$ Department of Agronomy, ${ }^{2}$ Department of Farm Power and Machinery, ${ }^{3}$ Department of Crop Physiology, Sindh Agriculture} University, Tandojam, Pakistan.

\begin{abstract}
Seed priming improves germination, crop stand and finally the yield. Two years field study was undertaken to assess the effect of primed seeds with different solutions and planting geometries on growth and yield of sunflower in agro-ecological conditions of Tandojam, Pakistan. The experiments were a randomized complete block design in factorial combination, replicated three times. An experimental unit was accommodated with three factors i.e. seed priming sources (No seed priming, seeds priming with canal water, seed primed with $1.0 \%$ Urea, and seed primed with $0.2 \% \mathrm{ZnSO}_{4}$ ), planting geometries (i.e. $55 \times 35 \mathrm{~cm}, 65 \times 30 \mathrm{~cm}, 75 \times 25 \mathrm{~cm}$, and $85 \times 20 \mathrm{~cm}$ ), and sunflower varieties (HO-1 and Hysun-33). Analysis of data revealed a significant effect of seed priming, geometry, varieties, and their interactions on sunflower growth and yield components. The highest seed yield $\left(\mathrm{kg} \mathrm{ha}^{-1}\right)$ and oil content (\%) were reported for seed primed with $0.2 \% \mathrm{ZnSO}_{4}$, followed by seeds primed with $1.0 \%$ Urea. In case of planting geometry, the maximum seed yield $\left(\mathrm{kg} \mathrm{ha}^{-1}\right)$ and oil content (\%) were noticed under planting geometry of $85 \times 20 \mathrm{~cm}$, followed by $75 \times 25 \mathrm{~cm}$. The variety HO-1 proved better in overall performance. The interaction of seed priming with $0.2 \% \mathrm{ZnSO}_{4}$ and planting geometry of $85 \times 20 \mathrm{~cm}$ produced the highest seed yield $\left(\mathrm{kg} \mathrm{ha}^{-1}\right)$ and oil content $(\%)$, followed by seed priming with $0.2 \% \mathrm{ZnSO}_{4}$ and $75 \times 25 \mathrm{~cm}$ planting geometry. It is concluded that sunflower yield could be enhanced substantially with seeds primed with $0.2 \% \mathrm{ZnSO}_{4}$ before sowing and arranged with planting geometry of $85 \mathrm{x} 20 \mathrm{~cm}$. The variety $\mathrm{HO}-1$ is relatively a better option for sunflower cultivation under agro-climatic conditions of Tandojam, Pakistan.

Received | August 14, 2019; Accepted | April 6, 2020; Published | April 25, 2020

*Correspondence | Zulfiqar Ali Abbasi, Department of Agronomy, Sindh Agriculture University, Tandojam, Pakistan; Email: zaabbasi786@ gmail.com

Citation | Abbasi, Z.A., M.N. Kandhro, A.A. Soomro, N. Leghari, M.I. Keerio, A.N. Shah and M.B. Abro. 2020. Nutri-seed priming and planting geometry effects on growth and oil production of sunflower varieties under the ecological conditions of Tandojam, Sindh, Pakistan. Sarhad Journal of Agriculture, 36(2): 447-458.

DOI | http://dx.doi.org/10.17582/journal.sja/2020/36.2.447.458

Keywords | Growth, Planting geometry, Seed priming, Sunflower, Varieties, Yield
\end{abstract}

\section{Introduction}

$\mathrm{E}$ dible oil has been reported as one of the most important commodities of daily use in Pakistan (Khan and Akmal, 2016; Amjad, 2014). The country is facing acute deficit of edible oil and hence imported annually about $88 \%$ edible oil for its consumption
(GoP, 2018). Pakistan is the $3^{\text {rd }}$ largest importer of edible oil in the world (Soomro, 2015). Sunflower is a major oilseed crop after soybean, palm and rapeseed in the world (Shah et al., 2013). It ranks $2^{\text {nd }}$ in Pakistan after cotton (Siddiqui, 2010). The crop is adapted well to the environmental conditions and prevailing cropping systems (Mahmood at al., 2018). In a 
study Bakhat et al. (2010) revealed that two seasons (spring and autumn) are suitable for the growing sunflower in Pakistan. During 2017-18, sunflower was cultivated on 82,186 hectares with production of 104,000 tones which yielded 40,000 tones oil (GoP, 2018). Buriro et al. (2015) reported that sunflower seed contains $47 \%$ oil, including vitamins A, D, E and $\mathrm{K}$ and linoleic acid. The sunflower oil consumption reduces the level of blood cholesterol (Kalaiyarasan and Vaiyapuri, 2007). The meal of sunflower seed is a good source of protein, used in livestock and poultry feed (Khalil and Jan, 2010). Fiber from the crop stem is used to make paper. Yellow dye is also obtained from flowers and purple black dye from seeds of sunflower (Tengong et al., 2010).

Priming of seeds is one of the important commercial processes, where germination rate and seedling stands in crops could be improved (Halmer, 2003). Seeds are kept in water or nutrient solution for certain period of time to regulate moisture or nutrient content, bringing them near to germination process and then sown in the field (Kouchebagh et al., 2014). The process involves advancing seed to a stage of germination, to enable fast and uniform emergence at planting (Dawal et al., 2017). Farming community can easily adopt many methods of seed priming for alleviating different stress levels. Furthermore, they can produce good quality seeds and use them for next crop cycle ultimately reducing costs of seed purchase (Chatterjee et al., 2018). Moeinzadeh et al. (2010) disclosed that seed priming caused improvement in vigour of sunflower. Priming of seeds with zinc could enhance metabolism of seeds and hence emergence and establishment of seedlings (Farooq et al., 2012).

Optimum plant density is a major concern in different crops (Akmal et al., 2014; Khan and Akmal, 2016). Planting of newly evolved sunflower varieties along with appropriate planting geometry is considered a reliable approach to increase production of edible oil for food security and reducing import bill (Zahoor et al., 2010). Ion et al. (2015) reported that optimum plant population depends on condition of crop growth. The findings of a study suggested that growth and yield traits of sunflower were superior under wider planting geometry $(75 \times 45 \mathrm{~cm})$ but yield was low due to inappropriate stand (Amjed et al., 2013). Considering importance of nutri-seed priming with planting geometry, this study was conducted for increasing yield of sunflower.

\section{Materials and Methods}

\section{Soil selection and experimental details}

Field experiment was conducted at Students' Experimental Farm of Sindh Agriculture University, Tandojam, Pakistan during autumn 2017 and repeated on same land during autumn 2018. The experimental design was a randomized complete block, factorial arrangements in three replications. The size of an experimental unit was $6 \mathrm{~m} \times 5 \mathrm{~m}\left(30 \mathrm{~m}^{2}\right)$. The soil of the experimental area was clay loam, which according to USDA system belongs to Order Aridisols and Subgroup Typic camborthids. The experimental field was ploughed two times with disc harrow, irrigated, dried to workable condition, levelled and finally seedbed was prepared by plowing with cultivator. The experiment consisted of four seed priming sources (No priming (seeds were not kept in water or nutrient solution), priming with canal water, priming with $1.0 \%$ Urea and priming with $0.2 \% \mathrm{ZnSO}_{4}$ ), four planting geometries (i.e. $55 \times 35,65 \times 30,75 \times 25$ and $85 \times$ $20 \mathrm{~cm}$ ), and two varieties (HO-1 and Hysun-33). The priming of seeds in canal water or nutrients solution was done in a plastic container at field for two hours and then seeds were sown immediately on same day in the field. For preparing 1.0\% Urea solution, $1 \mathrm{~kg}$ of Urea fertilizer was dissolved in 100 liters' water whereas for preparing $0.2 \%$ zinc concentration, $200 \mathrm{~g}$ of $\mathrm{ZnSO}_{4}$ were dissolved in 100 liters water. In $1^{\text {st }}$ year of experiment, priming of seeds was done on August 01, 2017 while in $2^{\text {nd }}$ year it was done on August 01, 2018. Sowing was done on same day of primed seed through single coulter hand drill. For maintaining proper plant to plant distance, thinning was done 15 days after sowing (DAS). The irrigation was applied keeping in view the soil condition and crop requirement. A total of five irrigations were applied at 15, 30, 45, 60 and 75 DAS. Each irrigation was applied to a level of farmer practice. The applications of N,P and $\mathrm{K}$ were 100, 50 and $50\left(\mathrm{~kg} \mathrm{ha}^{-1}\right)$ from Urea, SSP and SOP sources, respectively. The application of $\mathrm{N}$ was in three equal splits; i.e. $1^{\text {st }}$ at planting time, $2^{\text {nd }}$ at $1^{\text {st }}$ irrigation (15 DAS) and $3^{\text {rd }}$ at $3^{\text {rd }}$ irrigation (45 DAS). All phosphorus and potassium were applied at the time of land preparation. Inter-culturing was done for controlling weeds before $1^{\text {st }}, 2^{\text {nd }}$ and $3^{\text {rd }}$ irrigations, respectively.

Soil of the experimental area was analyzed before sowing and after harvesting (Table 1). Soil samples were taken with the help of soil auger at a depth of 
0-20 cm from five locations of total experimental area before sowing and after harvesting of the crop. The soil samples were air-dried, ground, sieved (2 mm) and placed in plastic containers. The samples were analyzed for various physical and chemical properties following the procedures of Ryan et al. (2001). Soil texture was measured by Bouyoucos hydrometer method. Electrical conductivity (EC) and soil $\mathrm{pH}$ was measured in 1:2 soil water extract using EC and pH meters, respectively. Walkley Black method was followed for the determination of organic matter content. Total $\mathrm{N}$ was calculated. However, soil was extracted for determining extractable $\mathrm{P}$ and $\mathrm{K}$ using Ammonium bicarbonate di-ethylene triamine penta acetic acid (AB-DTPA).

Table 1: Average physico-chemical properties experimental soil for the year 2017 and 2018.

$\begin{array}{lll}\text { Parameters } & \text { Before sowing } & \text { After } \\ \text { Soil texture } & & \\ \text { \%Sand } & 19.5 & - \\ \text { \%Silt } & 42.0 & - \\ \text { \%Clay } & 38.5 & - \\ \text { Textural class } & \text { Silty clay loam } & \\ \text { Soil chemical analysis } & & \\ \text { Soil pH } & 8.70 & 8.40 \\ \text { Organic matter (\%) } & 0.49 & 0.40 \\ \text { EC }\left(\mathrm{dS} \mathrm{m} \mathrm{m}^{-1}\right) & 2.36 & 1.50 \\ \text { Available } \mathrm{P}\left(\mathrm{mg} \mathrm{kg}^{-1}\right) & 1.30 & 0.90 \\ \text { Extractable K }\left(\mathrm{mg} \mathrm{kg}^{-1}\right) & 209 & 170 \\ \text { Total soil N }(\%) & 0.02 & 0.02\end{array}$

\section{Crop observations and measurement}

The agronomic and physiological observations were recorded for parameters of economic importance such as seed germination $\left(\mathrm{m}^{-2}\right)$, leaf area index (\%), crop growth rate $\left(\mathrm{g} \mathrm{m}^{-2} \mathrm{~d}^{-1}\right)$, plant height $(\mathrm{cm})$, head diameter $(\mathrm{cm})$, seeds head ${ }^{-1}$, seed weight head ${ }^{-1}(\mathrm{~g})$, 1000-seed weight $(\mathrm{g})$, biological yield $\left(\mathrm{kg} \mathrm{ha}^{-1}\right)$, seed yield $\left(\mathrm{kg} \mathrm{ha}^{-1}\right)$, harvest index (\%) and oil content (\%). Seed germination per square meter was noted by counting the emerged seedlings at 10 days after sowing. Leaf area index (\%) was measured at peak vegetative growth from randomly selected five plants by the formula: Leaf area plant ${ }^{-1}\left(\mathrm{~cm}^{2}\right) \div$ Ground area plant $\mathrm{t}^{1}\left(\mathrm{~cm}^{2}\right)$. Crop growth rate $\left(\mathrm{g} \mathrm{m}^{-2} \mathrm{~d}^{-1}\right)$ was recorded from five tagged plants using the formula: (W2 -W1) $\div(\mathrm{T} 2-\mathrm{T} 1)$ at peak vegetative growth. Plant height (cm) was measured with the help of measuring tape from base to the top at maturity from five selected plants. Head diameter $(\mathrm{cm})$ was noticed with the help of measuring tape at maturity from five tagged plants. Seeds head ${ }^{-1}$ were counted from five selected plants. Seed weight head ${ }^{-1}(\mathrm{~g})$ was noted from five tagged plants by weighing seeds on digital balance. Seed index (g) was recorded by weighing 1000 seeds on digital balance obtained from five selected plants. Biological yield $\left(\mathrm{kg} \mathrm{a}^{-1}\right)$ was noted by averaging the biomass weight of five tagged plants added to the biological yield of net experimental area of plot to estimate the biological yield per hectare according to the formula: Biological yield plot $^{-1}(\mathrm{~kg}) \div$ Area plot $^{-1}$ $\left(\mathrm{m}^{2}\right) \times 10000$. Seed yield $\left(\mathrm{kg} \mathrm{ha}^{-1}\right)$ was recorded from plants harvested for biological yield. The seeds of selected plants were shelled from head, sun dried and weighed using the formula: $=$ Seed yield plot $^{-1}(\mathrm{~kg})$ $\div$ Area plot $^{-1}\left(\mathrm{~m}^{2}\right) \times 10000$. Harvest index (\%) was calculated as per the formula: Seed yield $\left(\mathrm{kg} \mathrm{ha}^{-1}\right) \div$ Biological yield $\left(\mathrm{kg} \mathrm{ha}^{-1}\right) \times 100$. Oil was extracted by using $10 \mathrm{~g}$ seed for each treatment through Soxhlete apparatus available at Oilseeds Research Institute, Agriculture Research Centre, Tandojam by adopting proper method. The oil content \% was calculated according to the formula: Weight of oil $(\mathrm{g}) \div$ Weight of seed sample $(\mathrm{g}) \times 100$. The pooled data of each parameter for both years (2017 and 2018) is given below in respective tables.

\section{Statistical analysis}

The data were statistically analyzed following ANOVA technique using software Statistix version 8.1 (Statistix, 2006). The least significant difference (LSD) test was used at alpha 0.05 for comparing differences of treatments.

\section{Meteorological data}

The meteorological data of Tandojam during experimental seasons of both years (2017 and 2018) were obtained from Meteorological Station, Tandojam. The details of meteorological data on monthly basis for August, September, October and November regarding average temperature $\left({ }^{\circ} \mathrm{C}\right)$, Rainfall $(\mathrm{mm})$ and humidity (\%) are presented in Figure 1.

\section{Results and Discussion}

\section{Seed germination $\left(m^{-2}\right)$}

Optimum germination is pre-requisite for the desired seedlings per unit area, which ensures high yield. The statistical analysis of the data showed a significant $(p \leq 0.05)$ effect on germination for seed priming, June 2020 | Volume 36 | Issue 2 | Page 449 
geometry and interaction of seed priming $\mathrm{x}$ geometry whereas non-significant $(p \geq 0.05)$ effect for varieties including interaction of seed priming $\mathrm{x}$ geometry, seed priming $\mathrm{x}$ varieties, geometry $\mathrm{x}$ varieties and seed priming $\mathrm{x}$ geometry $\mathrm{x}$ varieties (Table 2). The seed priming with $0.2 \% \mathrm{ZnSO}_{4}$ gave higher seed germination followed by seed priming with $1.0 \%$ Urea. In case of planting geometry, highest seed germination was recorded in the geometry of $85 \times 20$ $\mathrm{cm}$ than all other planting geometries followed by 75 $\mathrm{x} 25 \mathrm{~cm}$. The possibility of improved seed germination under priming with zinc might be due to synthesis of Auxin in seeds (Rashid and Memon, 2001). The highest seed germination under the geometry 85 x $20 \mathrm{~cm}$ may be associated with optimum row and plant spacing. Previously, in a study, the population of sunflower plants was affected by planting densities, being higher in narrow while lower in wider row spacing (Khan and Akmal, 2016).
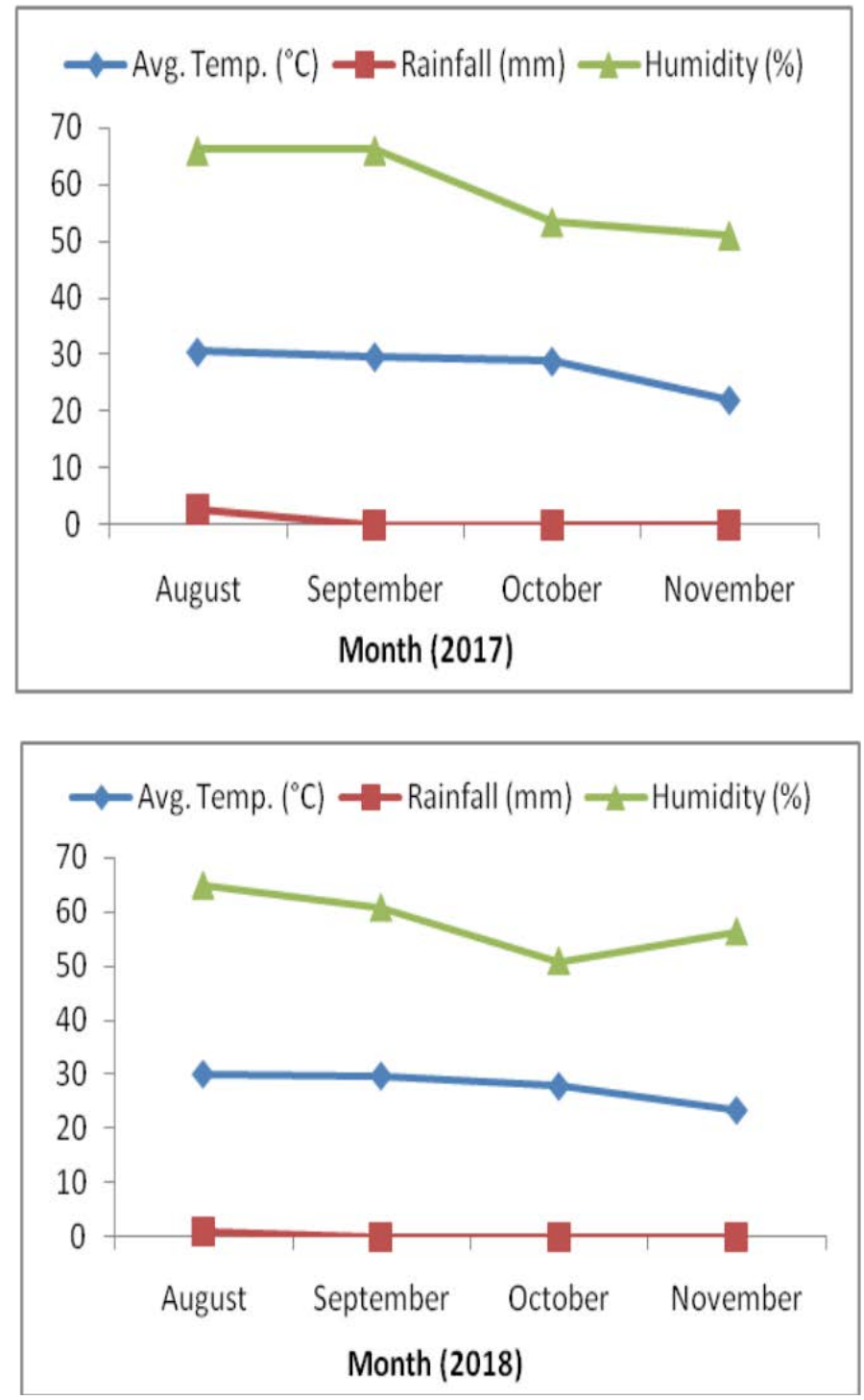

Figure 1: Meteorological data of Tandojam for crop growth season of sunflower during 2017 and 2018.
Table 2: Seed germination $\left(\mathrm{m}^{-2}\right)$ of different varieties of sunflower as influenced by seed priming and planting geometry.

\begin{tabular}{|c|c|c|c|c|c|c|}
\hline \multirow{2}{*}{$\begin{array}{l}\text { Varie- } \\
\text { ties }\end{array}$} & \multirow{2}{*}{$\begin{array}{l}\text { Seed priming } \\
\text { sources }\end{array}$} & \multicolumn{4}{|c|}{ Planting geometry $(\mathrm{cm})$} & \multirow[t]{2}{*}{ Mean } \\
\hline & & $55 \times 35$ & $65 \times 30$ & $75 \times 25$ & $85 \times 20$ & \\
\hline \multirow[t]{5}{*}{$\mathrm{HO}-1$} & No priming & 4.7 & 4.7 & 4.7 & 4.7 & 4.7 \\
\hline & Canal water & 5.3 & 5.7 & 6.0 & 6.7 & 5.9 \\
\hline & $1.0 \%$ Urea & 5.7 & 6.0 & 6.7 & 7.0 & 6.4 \\
\hline & $0.2 \% \mathrm{ZnSO}_{4}$ & 6.0 & 6.7 & 7.0 & 7.7 & 6.9 \\
\hline & Mean & 5.4 & 5.8 & 6.1 & 6.5 & 6.0 \\
\hline \multirow{5}{*}{$\begin{array}{l}\text { Hys- } \\
\text { un-33 }\end{array}$} & No priming & 4.7 & 4.7 & 4.7 & 4.7 & 4.7 \\
\hline & Canal water & 5.3 & 5.7 & 6.0 & 6.7 & 5.9 \\
\hline & $1.0 \%$ Urea & 5.7 & 6.0 & 6.7 & 7.0 & 6.4 \\
\hline & $0.2 \% \mathrm{ZnSO}_{4}$ & 6.0 & 6.7 & 7.0 & 7.7 & 6.9 \\
\hline & Mean & 5.4 & 5.8 & 6.1 & 6.5 & 6.0 \\
\hline \multirow{5}{*}{$\begin{array}{l}\text { Aver- } \\
\text { ages }\end{array}$} & No priming & 4.7 & 4.7 & 4.7 & 4.7 & $4.7 \mathrm{c}$ \\
\hline & Canal water & 5.3 & 5.7 & 6.0 & 6.7 & $5.9 \mathrm{~b}$ \\
\hline & $1.0 \%$ Urea & 5.7 & 6.0 & 6.7 & 7.0 & $6.4 \mathrm{~b}$ \\
\hline & $0.2 \% \mathrm{ZnSO}_{4}$ & 6.0 & 6.7 & 7.0 & 7.7 & $6.9 \mathrm{a}$ \\
\hline & Mean & $5.4 \mathrm{c}$ & $5.8 \mathrm{~b}$ & $6.1 \mathrm{~b}$ & $6.5 \mathrm{a}$ & \\
\hline \multicolumn{2}{|c|}{ Variables } & \multicolumn{2}{|l|}{ SE } & \multicolumn{2}{|l|}{$\mathrm{p}$-value } & LSD 5\% \\
\hline \multicolumn{2}{|c|}{ Priming $(\mathrm{P})$} & \multicolumn{2}{|l|}{0.1643} & \multicolumn{2}{|l|}{0.0288} & 0.3284 \\
\hline \multicolumn{2}{|c|}{ Geometry (G) } & \multicolumn{2}{|l|}{0.1643} & \multicolumn{2}{|l|}{0.0000} & 0.3284 \\
\hline \multicolumn{2}{|c|}{ Varieties (V) } & \multicolumn{2}{|l|}{0.1162} & \multicolumn{2}{|l|}{0.7211} & 0.2322 \\
\hline \multicolumn{2}{|l|}{$\mathrm{P} \times \mathrm{G}$} & \multicolumn{2}{|l|}{0.3286} & \multicolumn{2}{|l|}{0.0029} & 0.6569 \\
\hline \multicolumn{2}{|l|}{$\mathrm{P} \times \mathrm{V}$} & \multicolumn{2}{|l|}{0.2324} & \multicolumn{2}{|l|}{0.9428} & 0.4645 \\
\hline \multicolumn{2}{|l|}{$\mathrm{G} \times \mathrm{V}$} & \multicolumn{2}{|l|}{0.2324} & \multicolumn{2}{|l|}{0.9428} & 0.4645 \\
\hline \multicolumn{2}{|c|}{$\mathrm{P} \times \mathrm{G} \times \mathrm{V}$} & \multicolumn{2}{|l|}{0.4647} & \multicolumn{2}{|l|}{0.9988} & 0.9289 \\
\hline
\end{tabular}

\section{Leaf area index (\%)}

Seed priming, planting geometry, varieties, and interaction of seed priming $\mathrm{x}$ varieties, geometry $\mathrm{x}$ varieties, seed priming $\mathrm{x}$ geometry, and seed priming $\mathrm{x}$ geometry $\mathrm{x}$ varieties caused a substantial $(p \leq 0.05)$ impact on leaf area index (Table 3). Data showed that seed priming with $0.2 \% \mathrm{ZnSO}_{4}$ resulted in considerably $(p \leq 0.05)$ greater leaf area index, followed by seed priming with $1.0 \%$ Urea. As regards planting geometry, significantly $(p<0.05)$ highest leaf area index was noticed under the geometry of $85 \times 20 \mathrm{~cm}$, followed by $75 \times 25 \mathrm{~cm}$. Variety HO-1 proved better in leaf area index in contrast to Hysun-33. The higher and statistically equal $(\mathrm{LSD} 5 \%=0.4758)$ leaf area index was reported in $0.2 \% \mathrm{ZnSO}_{4}$ priming with 85 x $20 \mathrm{~cm}$ and $0.2 \% \mathrm{ZnSO}_{4}$ priming with $75 \times 25 \mathrm{~cm}$. Greatest leaf area index was noted in the integration of $\mathrm{HO}-1 \mathrm{x}$ priming with $0.2 \% \mathrm{ZnSO}_{4}$. The interaction of variety HO- 1 x geometry of $85 \times 20 \mathrm{~cm}$ produced highest leaf area-index. The maximum leaf areas 
index was observed in the interaction of $0.2 \% \mathrm{ZnSO}_{4}$ $\mathrm{x}$ geometry of $85 \times 20 \mathrm{~cm}$ x variety $\mathrm{HO}-1$. The better leaf area index with zinc priming may be attributed to active role of zinc for several enzymes including carbonic anhydrates, alcohol dehydrogenises and RNA polymerase (Rehman et al., 2015). The highest leaf area index under the geometry of $85 \times 20 \mathrm{~cm}$ may be associated to greatest growth of plant due to optimum row and plant spacing. Mehrpouyan et al. (2010) suggested the positive impact of planting densities on leaf area index of sunflower.

Table 3: Leaf area index (\%) of two varieties of sunflower as influenced by seed priming and planting geometry.

\begin{tabular}{|c|c|c|c|c|c|}
\hline \multirow{2}{*}{$\begin{array}{l}\text { Varie- } \\
\text { ties }\end{array}$} & \multirow{2}{*}{$\begin{array}{l}\text { Seed prim- } \\
\text { ing Sources }\end{array}$} & \multicolumn{3}{|c|}{ Planting geometry $(\mathrm{cm})$} & \multirow[t]{2}{*}{ Mean } \\
\hline & & $55 \times 3565 \times 30$ & $75 \times 25$ & $85 \times 20$ & \\
\hline \multirow[t]{5}{*}{$\mathrm{HO}-1$} & No priming & $10.8 \mathrm{n} 11.1 \mathrm{mn}$ & $12.0 \mathrm{kl}$ & $12.6 \mathrm{k}$ & 11. \\
\hline & Canal water & $13.7 \mathrm{j} \quad 16.7 \mathrm{f}$ & $17.1 \mathrm{ef}$ & $17.8 \mathrm{e}$ & 16.3 \\
\hline & $1.0 \%$ Urea & $15.7 \mathrm{~g} 18.9 \mathrm{~d}$ & $19.1 \mathrm{~cd}$ & $19.5 \mathrm{bcd}$ & 18.3 \\
\hline & $0.2 \% \mathrm{ZnSO}_{4}$ & 17.8 e $19.7 \mathrm{bc}$ & $20.1 \mathrm{~b}$ & $21.1 \mathrm{a}$ & 197 \\
\hline & Mean & $14.5 \mathrm{~d} 16.6 \mathrm{c}$ & $17.1 \mathrm{~b}$ & $17.8 \mathrm{a}$ & 10.3 \\
\hline \multirow{5}{*}{$\begin{array}{l}\text { Hys- } \\
\text { un-33 }\end{array}$} & No priming & $8.8 \mathrm{p} \quad 9.2 \mathrm{op}$ & 9.8 o & $10.8 \mathrm{n}$ & $9.7 \mathrm{~g}$ \\
\hline & Canal water & $10.7 \mathrm{n} 11.9 \mathrm{kl}$ & $11.81 \mathrm{~m}$ & $11.9 \mathrm{kl}$ & 11.6 \\
\hline & $1.0 \%$ Urea & $12.7 \mathrm{k} 14.0 \mathrm{ij}$ & 14.6 hi & $14.9 \mathrm{gh}$ & 14.1 \\
\hline & $0.2 \% \mathrm{ZnSO}_{4}$ & $14.1 \mathrm{ij} 15.5 \mathrm{~g}$ & $20.1 \mathrm{~b}$ & $17.1 \mathrm{ef}$ & 0.1 \\
\hline & Mean & $11.6 \mathrm{~g} 12.7 \mathrm{f}$ & $14.1 \mathrm{e}$ & $13.7 \mathrm{e}$ & 13.0 \\
\hline \multirow[t]{5}{*}{ Averages } & No priming & $9.8 \mathrm{k} \quad 10.2 \mathrm{k}$ & $10.9 \mathrm{j}$ & $11.7 \mathrm{i}$ & .0 \\
\hline & Canal water & $12.2 \mathrm{i} \quad 14.3 \mathrm{gh}$ & $14.5 \mathrm{gh}$ & $14.9 \mathrm{~g}$ & 14.0 \\
\hline & $1.0 \%$ Urea & $14.2 \mathrm{~h} 16.5 \mathrm{ef}$ & $16.9 \mathrm{de}$ & $17.2 \mathrm{~cd}$ & 16.2 \\
\hline & $0.2 \% \mathrm{ZnSO}_{4}$ & $16.0 \mathrm{f} 17.6 \mathrm{c}$ & $20.1 \mathrm{a}$ & $19.1 \mathrm{~b}$ & 18. \\
\hline & Mean & $13.0 \mathrm{c} 14.6 \mathrm{~b}$ & $15.6 \mathrm{a}$ & $15.7 \mathrm{a}$ & - \\
\hline \multicolumn{2}{|c|}{ Variables } & \multicolumn{2}{|l|}{ SE $\quad p$-value } & \multicolumn{2}{|l|}{ LSD 5\% } \\
\hline \multicolumn{2}{|c|}{ Priming $(\mathrm{P})$} & \multicolumn{2}{|l|}{0.16830 .0000} & \multicolumn{2}{|l|}{0.3364} \\
\hline \multicolumn{2}{|c|}{ Geometry (G) } & \multicolumn{2}{|l|}{0.11900 .0000} & \multicolumn{2}{|l|}{0.2379} \\
\hline \multicolumn{2}{|c|}{ Varieties (V) } & \multicolumn{2}{|l|}{0.16830 .0000} & \multicolumn{2}{|l|}{0.3364} \\
\hline \multicolumn{2}{|l|}{$P \times G$} & \multicolumn{2}{|l|}{0.23800 .0001} & \multicolumn{2}{|l|}{0.4758} \\
\hline \multicolumn{2}{|l|}{$\mathrm{P} \times \mathrm{V}$} & \multicolumn{2}{|l|}{0.33660 .0001} & \multicolumn{2}{|l|}{0.6729} \\
\hline \multicolumn{2}{|l|}{$\mathrm{G} \times \mathrm{V}$} & \multicolumn{2}{|l|}{0.23800 .0014} & \multicolumn{2}{|l|}{0.4758} \\
\hline \multicolumn{2}{|c|}{$\mathrm{P} \times \mathrm{G} \times \mathrm{V}$} & \multicolumn{2}{|l|}{0.16830 .0000} & \multicolumn{2}{|l|}{0.3364} \\
\hline
\end{tabular}

\section{Crop growth rate $\left(g m^{-2} d^{-1}\right)$}

Crop growth rate was significantly $(p \leq 0.05)$ impacted by seed priming, geometry and varieties including interaction of seed priming $\mathrm{x}$ varieties, geometry $\mathrm{x}$ varieties whereas, non-substantially $(p>0.05)$ by combination of seed priming $\mathrm{x}$ geometry and seed priming $\mathrm{x}$ geometry $\mathrm{x}$ varieties (Table 4 ). The results demonstrated that seed priming with $0.2 \% \mathrm{ZnSO}_{4}$ produced greater crop growth rate followed by seed priming with $1.0 \%$ Urea. With respect to planting geometry, highest crop growth rate was recorded in the planting geometry of $85 \times 20 \mathrm{~cm}$ followed by $75 \times 25 \mathrm{~cm}$. Among varieties, HO-1 proved better in crop growth rates as compared to Hysun-33. The interaction of $\mathrm{HO}-1 \mathrm{x}$ seed priming with $0.2 \% \mathrm{ZnSO}_{4}$ resulted in higher crop growth. The interaction of variety HO-1 with geometry $85 \times 20 \mathrm{~cm}$ produced greatest crop growth rate. The enhanced crop growth in zinc primed plots may be associated with active role of zinc for several enzymes (Rehman et al., 2015). The optimum row and plant spacing was the possible cause of higher crop growth rate under the geometry $85 \times 20 \mathrm{~cm}$. Jafri et al. (2015) concluded that seed priming caused boost in growth of sunflower.

Table 4: Crop growth rate $\left(g m^{-2} d^{-1}\right)$ of two varieties of sunflower as influenced by seed priming and planting geometry.

\begin{tabular}{|c|c|c|c|c|c|c|}
\hline \multirow{2}{*}{$\begin{array}{l}\text { Varie- } \\
\text { ties }\end{array}$} & \multirow{2}{*}{$\begin{array}{l}\text { Seed prim- } \\
\text { ing sources }\end{array}$} & \multicolumn{4}{|c|}{ Planting geometry $(\mathrm{cm})$} & \multirow[t]{2}{*}{ Mean } \\
\hline & & $55 \times 35$ & $65 \times 30$ & $75 \times 25$ & $85 \times 20$ & \\
\hline \multirow[t]{5}{*}{$\mathrm{HO}-1$} & No priming & 0.6 & 0.6 & 0.7 & 0.8 & $0.7 \mathrm{f}$ \\
\hline & Canal water & 1.4 & 1.3 & 1.5 & 2.1 & $1.6 \mathrm{~d}$ \\
\hline & $1.0 \%$ Urea & 1.5 & 2.9 & 3.0 & 3.1 & $2.6 \mathrm{~b}$ \\
\hline & $0.2 \% \mathrm{ZnSO}_{4}$ & 2.5 & 3.1 & 3.7 & 4.0 & $3.3 \mathrm{a}$ \\
\hline & Mean & $1.5 \mathrm{e}$ & $2.0 \mathrm{c}$ & $2.2 \mathrm{~b}$ & $2.5 \mathrm{a}$ & $2.1 \mathrm{a}$ \\
\hline \multirow{5}{*}{$\begin{array}{l}\text { Hys- } \\
\text { un-33 }\end{array}$} & No priming & 0.4 & 0.5 & 0.4 & 0.7 & $0.5 \mathrm{f}$ \\
\hline & Canal water & 1.1 & 1.0 & 1.2 & 1.2 & $1.1 \mathrm{e}$ \\
\hline & $1.0 \%$ Urea & 1.4 & 2.1 & 2.7 & 2.9 & $2.3 \mathrm{c}$ \\
\hline & $0.2 \% \mathrm{ZnSO}_{4}$ & 1.9 & 2.9 & 2.9 & 3.0 & $2.7 \mathrm{~b}$ \\
\hline & Mean & $1.2 \mathrm{f}$ & $1.6 \mathrm{de}$ & $1.8 \mathrm{~d}$ & $2.0 \mathrm{c}$ & $1.6 \mathrm{~b}$ \\
\hline \multirow{5}{*}{$\begin{array}{l}\text { Aver- } \\
\text { ages }\end{array}$} & No priming & 0.5 & 0.6 & 0.6 & 0.8 & $0.6 \mathrm{~d}$ \\
\hline & Canal water & 1.3 & 1.2 & 1.4 & 1.7 & $1.4 \mathrm{c}$ \\
\hline & $1.0 \%$ Urea & 1.5 & 2.5 & 2.9 & 3.0 & $2.5 \mathrm{~b}$ \\
\hline & $0.2 \% \mathrm{ZnSO}_{4}$ & 2.2 & 3.0 & 3.3 & 3.5 & $3.0 \mathrm{a}$ \\
\hline & Mean & $1.4 \mathrm{~d}$ & $1.8 \mathrm{c}$ & $2.0 \mathrm{~b}$ & $2.2 \mathrm{a}$ & - \\
\hline \multicolumn{2}{|c|}{ Variables } & \multicolumn{2}{|l|}{$\mathrm{SE}$} & \multicolumn{2}{|c|}{$\mathrm{p}$-value } & LSD 50 \\
\hline \multicolumn{2}{|c|}{ Priming $(\mathrm{P})$} & \multicolumn{2}{|l|}{0.1243} & \multicolumn{2}{|c|}{0.0499} & 0.2485 \\
\hline \multicolumn{2}{|c|}{ Geometry (G) } & \multicolumn{2}{|l|}{0.0879} & \multicolumn{2}{|c|}{0.0029} & 0.1757 \\
\hline \multicolumn{2}{|c|}{ Varieties $(V)$} & \multicolumn{2}{|l|}{0.1243} & \multicolumn{2}{|c|}{0.0000} & 0.2485 \\
\hline \multicolumn{2}{|l|}{$P \times G$} & \multicolumn{2}{|l|}{0.1758} & \multicolumn{2}{|c|}{0.3660} & - \\
\hline \multicolumn{2}{|l|}{$\mathrm{P} \times \mathrm{V}$} & \multicolumn{2}{|l|}{0.2487} & \multicolumn{2}{|c|}{0.0397} & 0.4971 \\
\hline \multicolumn{2}{|l|}{$\mathrm{G} \times \mathrm{V}$} & \multicolumn{2}{|l|}{0.1758} & \multicolumn{2}{|c|}{0.1597} & - \\
\hline \multicolumn{2}{|c|}{$\mathrm{P} \times \mathrm{G} \times \mathrm{V}$} & \multicolumn{2}{|l|}{0.3517} & \multicolumn{2}{|c|}{0.5225} & - \\
\hline
\end{tabular}

\section{Plant height $(\mathrm{cm})$}

A considerable $(p \leq 0.05)$ influence on plant height was noted for seed priming, geometry, varieties including interaction of varieties $\mathrm{x}$ geometry, and seed priming $\mathrm{x}$ geometry whereas, non-significant $(p>0.05)$ for interaction of seed priming $\mathrm{x}$ varieties, 
and seed priming $\mathrm{x}$ geometry $\mathrm{x}$ varieties (Table 5). The seed priming with $0.2 \% \mathrm{ZnSO}_{4}$ produced tallest plants, followed by seed priming with $1.0 \%$ Urea. In case of geometry, greatest plant height was recorded in the geometry of $85 \times 20 \mathrm{~cm}$, followed by geometry $75 \times 25 \mathrm{~cm}$. Variety HO-1 gave higher plant height against Hysun-33. The interaction of HO-1 with geometry of $85 \times 20 \mathrm{~cm}$ showed tallest plants. The integration of seed priming with $0.2 \% \mathrm{ZnSO}_{4}$ and $85 \times 20 \mathrm{~cm}$ resulted in greater plant height. Rehman et al. (2015) suggested that higher plant height in zinc primed treatments may be attributed to active role of zinc for several enzymes including carbonic anhydrates, alcohol dehydrogenases and RNA polymerase. The tall plants in geometry of $85 \times 20 \mathrm{~cm}$ were probably due to greater growth under optimum row and plant spacing. In another study, planting geometry caused significant effect on the height of plants (Vijayaakshmi et al., 2017).

Table 5: Plant height $(\mathrm{cm})$ of different varieties of sunflower as influenced by seed priming and planting geometry.

\begin{tabular}{|c|c|c|c|c|c|c|}
\hline \multirow[t]{2}{*}{ Varieties } & \multirow{2}{*}{$\begin{array}{l}\text { Seed prim- } \\
\text { ing sources }\end{array}$} & \multicolumn{4}{|c|}{ Planting geometry $(\mathrm{cm})$} & \multirow[t]{2}{*}{ Mean } \\
\hline & & $55 \times 35$ & $65 \times 30$ & $75 \times 25$ & $85 \times 20$ & \\
\hline \multirow[t]{5}{*}{ HO-1 } & No priming & 144 & 155 & 167 & 179 & 161 \\
\hline & Canal water & 168 & 176 & 184 & 188 & 179 \\
\hline & $1.0 \%$ Urea & 185 & 196 & 198 & 200 & 195 \\
\hline & $0.2 \% \mathrm{ZnSO}_{4}$ & 200 & 202 & 206 & 208 & 204 \\
\hline & Mean & $174 \mathrm{~d}$ & $182 \mathrm{c}$ & $189 \mathrm{~b}$ & $194 \mathrm{a}$ & $185 \mathrm{~A}$ \\
\hline \multirow{5}{*}{$\begin{array}{l}\text { Hys- } \\
\text { un-33 }\end{array}$} & No priming & 127 & 132 & 137 & 143 & 135 \\
\hline & Canal water & 148 & 152 & 156 & 163 & 154 \\
\hline & $1.0 \%$ Urea & 164 & 167 & 169 & 171 & 168 \\
\hline & $0.2 \% \mathrm{ZnSO}_{4}$ & 173 & 174 & 175 & 177 & 175 \\
\hline & Mean & $153 \mathrm{~h}$ & $156 \mathrm{~g}$ & $159 \mathrm{f}$ & 163 e & $158 \mathrm{~B}$ \\
\hline \multirow[t]{5}{*}{ Averages } & No priming & $135 \mathrm{k}$ & $144 \mathrm{j}$ & $152 \mathrm{i}$ & $161 \mathrm{gh}$ & $148 \mathrm{~d}$ \\
\hline & Canal water & $158 \mathrm{~h}$ & $164 \mathrm{~g}$ & $170 \mathrm{f}$ & 175 e & $167 \mathrm{c}$ \\
\hline & $1.0 \%$ Urea & $175 \mathrm{e}$ & $182 \mathrm{~d}$ & $183 \mathrm{~cd}$ & $185 \mathrm{~cd}$ & $181 \mathrm{~b}$ \\
\hline & $0.2 \% \mathrm{ZnSO}_{4}$ & $187 \mathrm{bc}$ & $188 \mathrm{bc}$ & $191 \mathrm{ab}$ & $193 \mathrm{a}$ & 189 a \\
\hline & Mean & $164 \mathrm{~d}$ & $169 \mathrm{c}$ & $174 \mathrm{~b}$ & $178 \mathrm{a}$ & - \\
\hline \multicolumn{2}{|l|}{ Variables } & \multicolumn{2}{|l|}{$\mathrm{SE}$} & \multicolumn{2}{|c|}{$\mathrm{p}$-value } & LSD 5\% \\
\hline \multicolumn{2}{|c|}{ Priming $(\mathrm{P})$} & \multicolumn{2}{|l|}{1.0574} & \multicolumn{2}{|l|}{0.0000} & 2.1137 \\
\hline \multicolumn{2}{|c|}{ Geometry (G) } & \multicolumn{2}{|l|}{1.0574} & \multicolumn{2}{|l|}{0.0000} & 2.1137 \\
\hline \multicolumn{2}{|c|}{ Varieties (V) } & \multicolumn{2}{|l|}{0.7477} & \multicolumn{2}{|l|}{0.0000} & 1.4946 \\
\hline \multicolumn{2}{|l|}{$P \times G$} & \multicolumn{2}{|l|}{2.1148} & \multicolumn{2}{|l|}{0.0000} & 4.2274 \\
\hline \multicolumn{2}{|l|}{$\mathrm{P} \times \mathrm{V}$} & \multicolumn{2}{|l|}{1.4954} & \multicolumn{2}{|l|}{0.1172} & 2.9893 \\
\hline \multicolumn{2}{|l|}{$G \times V$} & \multicolumn{2}{|l|}{1.4954} & \multicolumn{2}{|l|}{0.0003} & 2.9893 \\
\hline \multicolumn{2}{|c|}{$P \times G \times V$} & \multicolumn{2}{|l|}{2.9908} & \multicolumn{2}{|l|}{0.2560} & 5.9785 \\
\hline
\end{tabular}

The yield potential of sunflower crop depends upon the size of head. It is evident from the data that seed priming, geometry, varieties, and interaction of seed priming $\mathrm{x}$ varieties showed a significant $(p \leq 0.05)$ effect on head diameter whereas, non-significant $(p \geq 0.05)$ effect for interaction of seed priming $\mathrm{x}$ geometry, geometry $\mathrm{x}$ varieties and seed priming $\mathrm{x}$ geometry $\mathrm{x}$ varieties was noticed (Table 6). The results indicated that seed priming with $0.2 \% \mathrm{ZnSO}_{4}$ gave highest head diameter, followed by seed priming with $1.0 \%$ Urea. The planting geometry of $85 \times 20 \mathrm{~cm}$ conferred greatest head diameter, followed by $75 \times 25$ $\mathrm{cm}$. HO-1 gave more head diameter in comparison to Hysun-33. The highest head diameter was recorded in the interaction of variety $\mathrm{HO}-1 \mathrm{x}$ seed priming with $0.2 \% \mathrm{ZnSO}_{4}$. Moeinzadeh et al. (2010) suggested that seed priming caused improvement in sunflower head diameter. Plant density caused substantial influence on sunflower head diameter in a recent study (Mokhtari and Moosavi, 2016).

Table 6: Head diameter $(\mathrm{cm})$ of different varieties of sunflower as influenced by seed priming and planting geometry.

\begin{tabular}{|c|c|c|c|c|c|c|}
\hline \multirow{2}{*}{$\begin{array}{l}\text { Varie- } \\
\text { ties }\end{array}$} & \multirow{2}{*}{$\begin{array}{l}\text { Seed prim- } \\
\text { ing sources }\end{array}$} & \multicolumn{4}{|c|}{ Planting geometry $(\mathrm{cm})$} & \multirow[t]{2}{*}{ Mean } \\
\hline & & $55 \times 35$ & $65 \times 30$ & $75 \times 25$ & $85 \times 20$ & \\
\hline \multirow[t]{5}{*}{$\mathrm{HO}-1$} & No priming & 23.1 & 23.7 & 24.0 & 25.3 & $24.0 \mathrm{e}$ \\
\hline & Canal water & 24.9 & 25.0 & 26.0 & 26.8 & $25.7 \mathrm{~cd}$ \\
\hline & $1.0 \%$ Urea & 26.0 & 26.2 & 27.8 & 28.2 & $27.1 \mathrm{~b}$ \\
\hline & $0.2 \% \mathrm{ZnSO}_{4}$ & 27.0 & 28.9 & 29.2 & 30.9 & $29.0 \mathrm{a}$ \\
\hline & Mean & 25.3 & 26.0 & 26.8 & 27.8 & $26.5 \mathrm{~A}$ \\
\hline \multirow{5}{*}{$\begin{array}{l}\text { Hys- } \\
\text { un-33 }\end{array}$} & No priming & 22.4 & 22.7 & 23.6 & 24.0 & $23.2 \mathrm{f}$ \\
\hline & Canal water & 23.0 & 24.0 & 25.0 & 25.1 & $24.3 \mathrm{e}$ \\
\hline & $1.0 \%$ Urea & 24.6 & 25.0 & 25.6 & 26.1 & $25.3 \mathrm{~d}$ \\
\hline & $0.2 \% \mathrm{ZnSO}_{4}$ & 25.8 & 26.0 & 26.6 & 27.0 & $26.4 \mathrm{bc}$ \\
\hline & Mean & 24.0 & 24.4 & 25.2 & 25.6 & $24.8 \mathrm{~B}$ \\
\hline \multirow{5}{*}{$\begin{array}{l}\text { Aver- } \\
\text { ages }\end{array}$} & No priming & 22.8 & 23.2 & 23.8 & 24.7 & $23.6 \mathrm{~d}$ \\
\hline & Canal water & 24.0 & 24.5 & 25.5 & 26.0 & $25.0 \mathrm{c}$ \\
\hline & $1.0 \%$ Urea & 25.3 & 25.6 & 26.7 & 27.2 & $26.2 \mathrm{~b}$ \\
\hline & $0.2 \% \mathrm{ZnSO}_{4}$ & 26.4 & 27.5 & 27.9 & 29.0 & $27.7 \mathrm{a}$ \\
\hline & Mean & $24.6 \mathrm{~d}$ & $25.2 \mathrm{c}$ & $26.0 \mathrm{~b}$ & $26.7 \mathrm{a}$ & - \\
\hline \multicolumn{2}{|c|}{ Variables } & \multicolumn{2}{|l|}{ SE } & \multicolumn{2}{|c|}{$\mathrm{p}$-value } & LSD 5\% \\
\hline \multicolumn{2}{|c|}{ Priming $(\mathrm{P})$} & \multicolumn{2}{|l|}{0.2690} & \multicolumn{2}{|l|}{0.0000} & 0.5377 \\
\hline \multicolumn{2}{|c|}{ Geometry (G) } & \multicolumn{2}{|l|}{0.2690} & \multicolumn{2}{|l|}{0.0000} & 0.5377 \\
\hline \multicolumn{2}{|c|}{ Varieties (V) } & \multicolumn{2}{|l|}{0.1902} & \multicolumn{2}{|l|}{0.0000} & 0.3802 \\
\hline \multicolumn{2}{|l|}{$P \times G$} & \multicolumn{2}{|l|}{0.5380} & \multicolumn{2}{|l|}{0.9859} & 1.0754 \\
\hline \multicolumn{2}{|l|}{$\mathrm{P} \times \mathrm{V}$} & \multicolumn{2}{|l|}{0.3804} & \multicolumn{2}{|l|}{0.0146} & 0.7604 \\
\hline \multicolumn{2}{|l|}{$G \times V$} & \multicolumn{2}{|l|}{0.3804} & \multicolumn{2}{|l|}{0.3371} & 0.7604 \\
\hline \multicolumn{2}{|c|}{$\mathrm{P} \times \mathrm{G} \times \mathrm{V}$} & \multicolumn{2}{|l|}{0.7608} & \multicolumn{2}{|l|}{0.7491} & 1.5209 \\
\hline
\end{tabular}


Seeds head

The number of seeds head ${ }^{-1}$ represents the yield of crop. The analysis of variance indicated a considerable $(p \leq 0.05)$ influence on seeds head ${ }^{-1}$ for seed priming, geometry, varieties including integration of seed priming $\mathrm{x}$ varieties, whereas non-substantial $(p \geq 0.05)$ effect for interaction of seed priming $\mathrm{x}$ geometry, geometry $\mathrm{x}$ varieties, and seed priming $\mathrm{x}$ geometry $\mathrm{x}$ varieties (Table 7 ). The data denoted that seed priming with $0.2 \% \mathrm{ZnSO}_{4}$ resulted in greatest seeds head ${ }^{-1}$, followed by seed priming with $1.0 \%$ Urea. The geometry of $85 \times 20 \mathrm{~cm}$ produced highest seeds head ${ }^{-1}$, followed by $75 \times 25 \mathrm{~cm}$. The variety HO-1 proved better by producing higher number of seeds head ${ }^{-1}$ in contrast to Hysun-33. The interaction of variety $\mathrm{HO}-1 \times$ seed priming with $0.2 \% \mathrm{ZnSO}_{4}$ gave greatest seeds number head ${ }^{-1}$. The increased number of seeds head ${ }^{-1}$ in zinc primed plots may be linked to active role of zinc for several enzymes (Rehman et al., 2015). The optimum plant spacing was the possible cause of higher seeds number head ${ }^{-1}$ in the geometry of $85 \times 20$ $\mathrm{cm}$. Positive impact of planting densities on seeds head ${ }^{-1}$ of sunflower was also reported by Hekmat (2013).

Table 7: Seeds head ${ }^{-1}$ of different varieties of sunflower as influenced by seed priming and planting geometry.

\begin{tabular}{|c|c|c|c|c|c|c|}
\hline \multirow{2}{*}{$\begin{array}{l}\text { Varie- } \\
\text { ties }\end{array}$} & \multirow{2}{*}{$\begin{array}{l}\text { Seed prim- } \\
\text { ing sources }\end{array}$} & \multicolumn{4}{|c|}{ Planting geometry $(\mathrm{cm})$} & \multirow[t]{2}{*}{ Mean } \\
\hline & & $55 \times 35$ & $65 \times 30$ & $75 \times 25$ & $85 \times 20$ & \\
\hline \multirow[t]{5}{*}{$\mathrm{HO}-1$} & No priming & 671 & $799 x$ & $852 x$ & 859 & $795 \mathrm{f}$ \\
\hline & Canal water & 933 & 981 & 1017 & 1077 & $1002 \mathrm{~cd}$ \\
\hline & $1.0 \%$ Urea & 1088 & 1161 & 1181 & 1241 & $1168 \mathrm{~b}$ \\
\hline & $0.2 \% \mathrm{ZnSO}_{4}$ & 1199 & 1278 & 1297 & 1302 & $1269 \mathrm{a}$ \\
\hline & Mean & $973 \mathrm{~b}$ & $1055 \mathrm{a}$ & $1087 \mathrm{a}$ & $1120 \mathrm{a}$ & $1059 a$ \\
\hline \multirow{5}{*}{$\begin{array}{l}\text { Hys- } \\
\text { un-33 }\end{array}$} & No priming & 626 & 734 & 774 & 793 & $732 \mathrm{f}$ \\
\hline & Canal water & 888 & 900 & 939 & 949 & $919 \mathrm{e}$ \\
\hline & $1.0 \%$ Urea & $908 x$ & 946 & 1009 & 1047 & $978 \mathrm{de}$ \\
\hline & $0.2 \% \mathrm{ZnSO}_{4}$ & 998 & 1071 & 1097 & 1102 & $1067 \mathrm{c}$ \\
\hline & Mean & $855 \mathrm{c}$ & $913 b \mathrm{c}$ & $955 \mathrm{x} \mathrm{b}$ & $973 \mathrm{~b}$ & $924 \mathrm{~b}$ \\
\hline \multirow{5}{*}{$\begin{array}{l}\text { Aver- } \\
\text { ages }\end{array}$} & No priming & 649 & 767 & 813 & 826 & $764 \mathrm{~d}$ \\
\hline & Canal water & 911 & 941 & 978 & 1013 & $961 \mathrm{c}$ \\
\hline & $1.0 \%$ Urea & 998 & 1054 & 1095 & 1144 & $1073 \mathrm{~b}$ \\
\hline & $0.2 \% \mathrm{ZnSO}_{4}$ & 1098 & 1174 & 1197 & 1202 & $1168 \mathrm{a}$ \\
\hline & Mean & $914 \mathrm{c}$ & $984 \mathrm{~b}$ & $1021 \mathrm{ab}$ & $1046 \mathrm{a}$ & - \\
\hline
\end{tabular}

$\begin{array}{llll}\text { Variables } & \text { SE } & \text { p-value } & \text { LSD 5\% } \\ \text { Priming }(\mathrm{P}) & 27.993 & 0.0000 & 55.957 \\ \text { Geometry }(\mathrm{G}) & 27.993 & 0.0001 & 55.957 \\ \text { Varieties }(\mathrm{V}) & 19.794 & 0.0000 & 39.568 \\ \mathrm{P} \times \mathrm{G} & 55.986 & 0.9727 & 111.91 \\ \mathrm{P} \times \mathrm{V} & 39.588 & 0.0272 & 79.135 \\ \mathrm{G} \times \mathrm{V} & 39.588 & 0.9571 & 79.135 \\ \mathrm{P} \times \mathrm{G} \times \mathrm{V} & 79.176 & 1.0000 & 158.27\end{array}$

Table 8: Seed weight head ${ }^{-1}(g)$ of different varieties of sunflower as influenced by seed priming and planting geometry.

\begin{tabular}{|c|c|c|c|c|c|c|}
\hline Varie- & & Plan & n & $\mathrm{cm}$ & & Mean \\
\hline & & $55 \times 35$ & $65 \times 30$ & $75 \times 25$ & $85 \times 20$ & \\
\hline $\mathrm{HO}-1$ & No priming & 63.1 & 81.9 & 87.9 & 84.7 & $79.4 \mathrm{f}$ \\
\hline & Canal water & 80.6 & 100.1 & 104.2 & 105.4 & $97.6 \mathrm{~d} \mathrm{e}$ \\
\hline & $1.0 \%$ Urea & 96.1 & 116.8 & 129.4 & 121.6 & $116.0 \mathrm{~b}$ \\
\hline & $0.2 \% \mathrm{ZnSO}_{4}$ & ${ }_{4} 114.7$ & 131.8 & 134.9 & 133.8 & $128.8 \mathrm{a}$ \\
\hline & Mean & $88.6 \mathrm{~d}$ & 107.7ab & $114.1 \mathrm{a}$ & $111.4 \mathrm{a}$ & $105.4 \mathrm{a}$ \\
\hline & No priming & 62.2 & 68.6 & 76.3 & 85.1 & $73.1 \mathrm{f}$ \\
\hline & Canal water & 76.3 & 96.7 & 98.7 & 101.5 & $93.3 \mathrm{e}$ \\
\hline & $1.0 \%$ Urea & 86.4 & 105.9 & 106.1 & 107.8 & $101.6 \mathrm{~d}$ \\
\hline & $0.2 \% \mathrm{ZnSO}_{4}$ & ${ }_{4} 105.3$ & 109.9 & 110.5 & 110.8 & $109.1 \mathrm{c}$ \\
\hline & Mean & $82.6 \mathrm{~d}$ & $95.3 \mathrm{c}$ & $97.9 \mathrm{c}$ & $101.3 \mathrm{bc}$ & $94.3 \mathrm{~b}$ \\
\hline Aver- & No priming & $62.7 \mathrm{j}$ & $75.3 \mathrm{i}$ & $80.5 \mathrm{hi}$ & $86.5 \mathrm{gh}$ & $76.2 \mathrm{~d}$ \\
\hline & Canal water & $78.5 \mathrm{hi}$ & $98.4 \mathrm{ef}$ & 102.1de & 10 & $95.4 \mathrm{c}$ \\
\hline & $1.0 \%$ Urea & $91.3 \mathrm{fg}$ & $111.4 \mathrm{bc}$ & $113.9 \mathrm{ab}$ & 118.6ab & $108.8 \mathrm{~b}$ \\
\hline & $0.2 \% \mathrm{ZnSO}_{4}$ & $\begin{array}{l}110.0 \\
\text { bcd }\end{array}$ & $120.9 \mathrm{a}$ & $122.2 \mathrm{a}$ & $122.9 \mathrm{a}$ & $119.0 \mathrm{a}$ \\
\hline & Mea & $85.6 \mathrm{c}$ & 10 & 104.6ab & 1 & - \\
\hline Varial & & $\mathrm{SE}$ & & $\mathrm{p}$-value & & LSD 5 \\
\hline Primin & $\lg (\mathrm{P})$ & 2.2771 & & 0.0000 & & 4.5519 \\
\hline Geor & etry $(G)$ & 2.2771 & & 0.0000 & & 4.5519 \\
\hline Varieti & ies $(V)$ & 1.6102 & & 0.0000 & & 3.2187 \\
\hline $\mathrm{P} \times \mathrm{G}$ & & 4.5542 & & 0.4996 & & 9.1037 \\
\hline $\mathrm{P} \times \mathrm{V}$ & & 3.2203 & & 0.0040 & & 6.4373 \\
\hline$G \times V$ & & 3.2203 & & 0.3373 & & 6.4373 \\
\hline$P \times G$ & $\times \mathrm{V}$ & 6.4406 & & 0.8572 & & 12.875 \\
\hline
\end{tabular}

\section{Seed weight head ${ }^{-1}(g)$}

A significant $(p \leq 0.05)$ effect on seed weight head ${ }^{-1}$ was noticed by seed priming, geometry and varieties including interaction of seed priming $\mathrm{x}$ varieties, geometry $\mathrm{x}$ varieties whereas, non-substantial $(p>0.05)$ effect was observed for seed priming $\mathrm{x}$ geometry and seed priming $\mathrm{x}$ geometry $\mathrm{x}$ varieties (Table 8). The results indicated that seed priming with $0.2 \% \mathrm{ZnSO}_{4}$ gave higher seed weight head ${ }^{1}$, followed by seed priming with $1.0 \%$ Urea. The highest seed weight head ${ }^{-1}$ was reported in geometry of $85 \times 20 \mathrm{~cm}$, followed by $75 \times 25 \mathrm{~cm}$. Among varieties, HO-1 surpassed Hysun-33 in seed weight head $^{-1}$. The greatest seed weight head ${ }^{-1}$ was found in the interaction of $0.2 \% \mathrm{ZnSO}_{4} \times$ geometry of $85 \times 20$ $\mathrm{cm}$. The combination of variety $\mathrm{HO}-1 \mathrm{x}$ seed priming with $0.2 \% \mathrm{ZnSO}_{4}$ produced highest seed weight head ${ }^{-}$ 1. The interaction of variety HO- $1 \mathrm{x}$ geometry of $85 \mathrm{x}$ $20 \mathrm{~cm}$ gave greatest seed weight head ${ }^{-1}$. The possibility 
of improved seed weight head ${ }^{-1}$ in priming with zinc might be due to synthesis of Auxin or indole acetic acid in seeds (Rashid and Memon, 2001). The highest seed weight head ${ }^{-1}$ with the geometry of $85 \times 20 \mathrm{~cm}$ may be associated to optimum row and plant spacing. Planting densities caused positively significant impact on sunflower seed weight head ${ }^{-1}$ (Rasool et al., 2015).

Table 9: 1000-seed weight (g) of different varieties of sunflower as influenced by seed priming and planting geometry.

\begin{tabular}{|c|c|c|c|c|c|c|}
\hline \multirow{2}{*}{$\begin{array}{l}\text { Varie- } \\
\text { ties }\end{array}$} & \multirow{2}{*}{$\begin{array}{l}\text { Seed prim- } \\
\text { ing sources }\end{array}$} & \multicolumn{4}{|c|}{ Planting geometry $(\mathrm{cm})$} & \multirow[t]{2}{*}{ Mean } \\
\hline & & $55 \times 35$ & $65 \times 30$ & $75 \times 25$ & $85 \times 20$ & \\
\hline \multirow[t]{5}{*}{$\mathrm{HO}-1$} & No priming & 66.7 & 67.7 & 68.3 & 68.7 & 67.8 \\
\hline & Canal water & 69.3 & 80.0 & 80.0 & 80.7 & 77.5 \\
\hline & $1.0 \%$ Urea & 72.7 & 81.0 & 81.7 & 83.3 & 79.7 \\
\hline & $0.2 \% \mathrm{ZnSO}_{4}$ & 75.0 & 81.7 & 90.0 & 91.7 & 84.6 \\
\hline & Mean & 70.9 & 77.6 & 80.0 & 81.1 & $77.4 \mathrm{~A}$ \\
\hline \multirow{5}{*}{$\begin{array}{l}\text { Hys- } \\
\text { un-33 }\end{array}$} & No priming & 64.0 & 65.1 & 66.3 & 66.7 & 65.5 \\
\hline & Canal water & 67.7 & 75.0 & 73.3 & 76.7 & 73.2 \\
\hline & $1.0 \%$ Urea & 70.0 & 78.3 & 75.0 & 78.3 & 75.4 \\
\hline & $0.2 \% \mathrm{ZnSO}_{4}$ & 73.3 & 80.0 & 85.0 & 88.3 & 81.7 \\
\hline & Mean & 68.8 & 74.6 & 74.9 & 77.5 & $73.9 \mathrm{~B}$ \\
\hline \multirow{5}{*}{$\begin{array}{l}\text { Aver- } \\
\text { ages }\end{array}$} & No priming & 65.4 & 66.4 & 67.3 & 67.7 & $66.7 \mathrm{~d}$ \\
\hline & Canal water & 68.5 & 77.5 & 76.7 & 78.7 & $75.3 \mathrm{c}$ \\
\hline & $1.0 \%$ Urea & 71.4 & 79.7 & 78.4 & 80.8 & $77.5 \mathrm{~b}$ \\
\hline & $0.2 \% \mathrm{ZnSO}_{4}$ & 74.2 & 80.9 & 87.5 & 90.0 & $83.1 \mathrm{a}$ \\
\hline & Mean & $69.8 \mathrm{~b}$ & $76.1 \mathrm{a}$ & $77.5 \mathrm{a}$ & $79.3 \mathrm{a}$ & - \\
\hline \multicolumn{2}{|c|}{ Variables } & \multicolumn{2}{|l|}{$\mathrm{SE}$} & \multicolumn{2}{|c|}{$\mathrm{p}$-value } & LSD 5\% \\
\hline \multicolumn{2}{|c|}{ Priming $(\mathrm{P})$} & \multicolumn{2}{|l|}{1.6099} & \multicolumn{2}{|l|}{0.0000} & 3.2181 \\
\hline \multicolumn{2}{|c|}{ Geometry (G) } & \multicolumn{2}{|l|}{1.6099} & \multicolumn{2}{|l|}{0.0000} & 3.2181 \\
\hline \multicolumn{2}{|c|}{ Varieties $(V)$} & \multicolumn{2}{|l|}{1.1383} & \multicolumn{2}{|l|}{0.0035} & 2.2755 \\
\hline \multicolumn{2}{|l|}{$P \times G$} & \multicolumn{2}{|l|}{3.2197} & \multicolumn{2}{|l|}{0.1546} & 6.4361 \\
\hline \multicolumn{2}{|l|}{$\mathrm{P} \times \mathrm{V}$} & \multicolumn{2}{|l|}{2.2767} & \multicolumn{2}{|l|}{0.9005} & 4.5510 \\
\hline \multicolumn{2}{|l|}{$\mathrm{G} \times \mathrm{V}$} & \multicolumn{2}{|l|}{2.2767} & \multicolumn{2}{|l|}{0.8302} & 4.5510 \\
\hline \multicolumn{2}{|c|}{$\mathrm{P} \times \mathrm{G} \times \mathrm{V}$} & \multicolumn{2}{|l|}{4.5534} & \multicolumn{2}{|l|}{0.9999} & 9.1021 \\
\hline
\end{tabular}

1000-seed weight $(g)$

Seed index is a key indicator to attain optimal weight of seeds. Seed priming, geometry and varieties showed a substantial $(p \leq 0.05)$ effect impact on seed index whereas, the interaction of seed priming $\mathrm{x}$ geometry, seed priming $\mathrm{x}$ varieties, geometry $\mathrm{x}$ varieties, and seed priming $\mathrm{x}$ geometry $\mathrm{x}$ varieties caused a nonsubstantial $(p>0.05)$ impact on seed index (Table 9). The seed priming with $0.2 \% \mathrm{ZnSO}_{4}$ conferred highest seed index, followed by seed priming with $1.0 \%$ Urea. The greatest seed index was noted in geometry of 85 x $20 \mathrm{~cm}$, followed by $75 \times 25 \mathrm{~cm}$. The variety HO-1 proved better in seed index as compared to Hysun-33.
The enhanced seed index with zinc priming may be attributed to active role of zinc for several enzymes including RNA polymerase, carbonic anhydrate and alcohol dehydrogenises (Rehman et al., 2015). Seed priming caused improvement in seed index (Jafri et al., 2015). The highest seed index in the geometry of $85 \times 20 \mathrm{~cm}$ may be associated to optimum plant growth. Kazemeini et al. (2009) suggested positive effect of planting density on seed index of sunflower.

Table 10: Biological yield ( $\mathrm{kg} \mathrm{ha}^{-1}$ ) of different varieties of sunflower as influenced by seed priming and planting geometry.

\begin{tabular}{|c|c|c|c|c|c|c|}
\hline \multirow{2}{*}{$\begin{array}{l}\text { Vari- } \\
\text { eties }\end{array}$} & \multirow{2}{*}{$\begin{array}{l}\text { Seed prim- } \\
\text { ing sources }\end{array}$} & \multicolumn{4}{|c|}{ Planting geometry $\mathrm{cm}$} & \multirow[t]{2}{*}{ Mean } \\
\hline & & $55 \times 35$ & $65 \times 30$ & $75 \times 25$ & $85 \times 20$ & \\
\hline \multirow{5}{*}{$\begin{array}{l}\mathrm{HO}- \\
1\end{array}$} & No priming & 6536 & 6623 & 6796 & 7035 & $6748 \mathrm{e}$ \\
\hline & Canal water & 7660 & 7350 & 7723 & 7903 & $7659 \mathrm{~d}$ \\
\hline & $1.0 \%$ Urea & 8483 & 10106 & 12523 & 12633 & $10936 \mathrm{~b}$ \\
\hline & $0.2 \% \mathrm{ZnSO}_{4}$ & 8583 & 11753 & 12640 & 12963 & $11485 \mathrm{a}$ \\
\hline & Mean & $7815 \mathrm{~d}$ & $8958 \mathrm{~b}$ & 9920 a & $10133 a$ & 9207 a \\
\hline \multirow{5}{*}{$\begin{array}{l}\text { Hys- } \\
\text { un-33 }\end{array}$} & No priming & 6516 & 6556 & 6728 & 6900 & $6675 \mathrm{e}$ \\
\hline & Canal water & 7346 & 7280 & 7436 & 7830 & $7473 \mathrm{~d}$ \\
\hline & $1.0 \%$ Urea & 8226 & 9856 & 10130 & 10133 & $9586 \mathrm{c}$ \\
\hline & $0.2 \% \mathrm{ZnSO}_{4}$ & 8313 & 10106 & 10393 & 10490 & $9825 \mathrm{c}$ \\
\hline & Mean & $7600 \mathrm{~d}$ & $8450 \mathrm{c}$ & $8672 \mathrm{bc}$ & 8838 b & $8390 \mathrm{~b}$ \\
\hline \multirow{5}{*}{$\begin{array}{l}\text { Aver- } \\
\text { ages }\end{array}$} & No priming & $6526 \mathrm{~h}$ & $6590 \mathrm{~h}$ & $6762 \mathrm{~h}$ & 6968gh & $6711 \mathrm{~d}$ \\
\hline & Canal water & 7503 ef & 73 & 7580 ef & 7866 e & $7566 \mathrm{c}$ \\
\hline & $1.0 \%$ Urea & $8355 \mathrm{~d}$ & $9981 c$ & $11326 \mathrm{ab}$ & $83 a$ & $10261 \mathrm{~b}$ \\
\hline & $0.2 \% \mathrm{ZnSO}_{4}$ & $8448 d$ & $10930 \mathrm{~b}$ & $11516 \mathrm{a}$ & $11726 a$ & $10655 \mathrm{a}$ \\
\hline & Mean & 7708 c & 8704 b & $9296 \mathrm{a}$ & 9486 a & - \\
\hline \multicolumn{2}{|c|}{ Variables } & $\mathrm{SE}$ & & $\mathrm{p}$-value & & LSD 5\% \\
\hline \multicolumn{2}{|c|}{ Priming $(\mathrm{P})$} & 116.57 & & 0.0000 & & 233.02 \\
\hline \multicolumn{2}{|c|}{ Geometry (G) } & 116.57 & & 0.0000 & & 233.02 \\
\hline \multicolumn{2}{|c|}{ Varieties (V) } & 82.427 & & 0.0000 & & 164.77 \\
\hline \multicolumn{2}{|l|}{$P \times G$} & 233.14 & & 0.0000 & & 466.04 \\
\hline \multicolumn{2}{|l|}{$\mathrm{P} \times \mathrm{V}$} & 164.85 & & 0.0000 & & 329.54 \\
\hline \multicolumn{2}{|l|}{$\mathrm{G} \times \mathrm{V}$} & 164.85 & & 0.0000 & & 329.54 \\
\hline \multicolumn{2}{|c|}{$\mathrm{P} \times \mathrm{G} \times \mathrm{V}$} & 329.71 & & 0.0004 & & 659.08 \\
\hline
\end{tabular}

\section{Biological yield $\left(\mathrm{kg} \mathrm{ha}^{-1}\right)$}

The analysis of variance indicated a considerable $(p \leq 0.05)$ influence on biological yield for seed priming, geometry, varieties, interaction of seed priming $\mathrm{x}$ varieties, geometry $\mathrm{x}$ varieties, seed priming $\mathrm{x}$ geometry, and seed priming $\mathrm{x}$ geometry $\mathrm{x}$ varieties (Table 10). The results revealed that seed priming with $0.2 \% \mathrm{ZnSO}_{4}$ resulted in greatest biological yield, followed by seed priming with $1.0 \%$ Urea. The highest biological yield was recorded in the geometry of 85 x $20 \mathrm{~cm}$, followed by $75 \times 25 \mathrm{~cm}$. Among varieties, 
HO-1 proved better in contrast to Hysun-33. The interaction of priming with $0.2 \% \mathrm{ZnSO}_{4} \mathrm{x}$ geometry of $85 \times 20 \mathrm{~cm}$ gave highest biological yield. The integration of variety $\mathrm{HO}-1$ with seed priming with $0.2 \% \mathrm{ZnSO}_{4}$ produced greatest biological yield. The interaction of HO- $1 \times$ geometry of $85 \times 20 \mathrm{~cm}$ gave highest biological yield. The enhanced biological yield in zinc primed plots may be due to active role of zinc for several enzymes (Rehman et al., 2015). Mrda et al. (2010) displayed that seed priming increased biological yield of sunflower plants. The higher biological yield in geometry of $85 \times 20 \mathrm{~cm}$ was perhaps due to optimum densities on biological yield of sunflower was reported by Tavakoli (2013).

Table 11: Seed yield $\left(\mathrm{kg} \mathrm{ha}^{-1}\right)$ of different varieties of sunflower as influenced by seed priming and planting geometry.

\begin{tabular}{|c|c|c|c|c|c|c|}
\hline \multirow{2}{*}{$\begin{array}{l}\text { Varie- } \\
\text { ties }\end{array}$} & \multirow{2}{*}{$\begin{array}{l}\text { Seed prim- } \\
\text { ing sources }\end{array}$} & \multicolumn{4}{|c|}{ Planting geometry $(\mathrm{cm})$} & \multirow[t]{2}{*}{ Mean } \\
\hline & & $55 \times 35$ & $65 \times 30$ & $75 \times 25$ & $85 \times 20$ & \\
\hline \multirow[t]{5}{*}{$\mathrm{HO}-1$} & No priming & 1800 & 1880 & 1886 & 1896 & $1865 \mathrm{~d}$ \\
\hline & Canal water & 1870 & 1913 & 1953 & 2036 & 1943 c \\
\hline & $1.0 \%$ Urea & 2200 & 2263 & 2528 & 2540 & $2383 \mathrm{~b}$ \\
\hline & $0.2 \% \mathrm{ZnSO}_{4}$ & 2286 & 2393 & 2550 & 2596 & $2456 \mathrm{a}$ \\
\hline & Mean & $2039 \mathrm{ef}$ & $2112 \mathrm{~cd}$ & $2229 \mathrm{ab}$ & 2267 a & $2162 \mathrm{a}$ \\
\hline \multirow{5}{*}{$\begin{array}{l}\text { Hys- } \\
\text { un-33 }\end{array}$} & No priming & 1773 & 1805 & 1813 & 1821 & $1803 \mathrm{~d}$ \\
\hline & Canal water & 1820 & 1876 & 1883 & 1896 & $1869 \mathrm{~d}$ \\
\hline & $1.0 \%$ Urea & 2160 & 2243 & 2468 & 2476 & $2337 \mathrm{~b}$ \\
\hline & $0.2 \% \mathrm{ZnSO}_{4}$ & 2260 & 2370 & 2475 & 2486 & $2397 \mathrm{ab}$ \\
\hline & Mean & $2003 f$ & 2073de & $2160 \mathrm{c}$ & $2170 \mathrm{bc}$ & $2101 \mathrm{~b}$ \\
\hline \multirow{5}{*}{$\begin{array}{l}\text { Aver- } \\
\text { ages }\end{array}$} & No priming & 1786 & 1842 & 1850 & 1858 & $1834 \mathrm{~d}$ \\
\hline & Canal water & 1845 & 1895 & 1918 & 1966 & $1906 \mathrm{c}$ \\
\hline & $1.0 \%$ Urea & 2180 & 2253 & 2498 & 2508 & $2360 \mathrm{~b}$ \\
\hline & $0.2 \% \mathrm{ZnSO}_{4}$ & 2273 & 2381 & 2512 & 2541 & 2427 a \\
\hline & Mean & $2021 c$ & $2093 b$ & $2194 \mathrm{a}$ & $2218 \mathrm{a}$ & - \\
\hline \multicolumn{2}{|c|}{ Variables } & \multicolumn{2}{|l|}{ SE } & \multicolumn{2}{|l|}{$\mathrm{p}$-value } & LSD 5\% \\
\hline \multicolumn{2}{|c|}{ Priming $(\mathrm{P})$} & \multicolumn{2}{|l|}{23.965} & \multicolumn{2}{|l|}{0.0000} & 47.905 \\
\hline \multicolumn{2}{|c|}{ Geometry (G) } & \multicolumn{2}{|l|}{23.965} & \multicolumn{2}{|l|}{0.0000} & 47.905 \\
\hline \multicolumn{2}{|c|}{ Varieties (V) } & \multicolumn{2}{|l|}{16.946} & \multicolumn{2}{|l|}{0.0007} & 33.874 \\
\hline \multicolumn{2}{|l|}{$P \times G$} & \multicolumn{2}{|l|}{47.929} & \multicolumn{2}{|l|}{0.0005} & 95.810 \\
\hline \multicolumn{2}{|l|}{$\mathrm{P} \times \mathrm{V}$} & \multicolumn{2}{|l|}{33.891} & \multicolumn{2}{|l|}{0.9491} & 67.748 \\
\hline \multicolumn{2}{|l|}{$\mathrm{G} \times \mathrm{V}$} & \multicolumn{2}{|l|}{33.891} & \multicolumn{2}{|l|}{0.5379} & 67.748 \\
\hline \multicolumn{2}{|c|}{$\mathrm{P} \times \mathrm{G} \times \mathrm{V}$} & \multicolumn{2}{|l|}{67.783} & \multicolumn{2}{|l|}{0.9994} & 135.50 \\
\hline
\end{tabular}

Seed yield $\left(\mathrm{kg} \mathrm{ha}^{-1}\right)$

Seed yield is a major decision used in agricultural crop. It refers as output produced per unit area. A significant $(p \leq 0.05)$ effect on seed yield was noticed for seed row and plant spacing. Positive impact of planting

priming, geometry, varieties and integration of seed priming $\mathrm{x}$ geometry, whereas non-substantial $(p \geq 0.05)$ effect was observed for the interaction of seed priming $\mathrm{x}$ varieties, geometry $\mathrm{x}$ varieties, and seed priming $\mathrm{x}$ geometry $\mathrm{x}$ varieties (Table 11). The results illustrated that highest seed yield was recorded in seed priming with $0.2 \% \mathrm{ZnSO}_{4}$, followed by seed priming with $1.0 \%$ Urea. The greatest and statistical similar seed yield was noted in the geometry of $85 \times 20 \mathrm{~cm}$ and $75 \times 25$. The variety $\mathrm{HO}-1$ produced highest seed yield as compared to Hysun-33. The interaction of seed priming with $0.2 \% \mathrm{ZnSO}_{4} \times$ geometry of $85 \times 20 \mathrm{~cm}$ resulted in highest seed yield. The possibility of improved seed yield in priming with zinc may be attributed to its active role for several enzymes including carbonic anhydrate, alcohol dehydrogenises and RNA polymerase (Rehman et al., 2015). The increased seed yield in the geometry of $85 \times 20 \mathrm{~cm}$ may be associated to optimum growth and population of plants. Positive impact of planting geometries on seed yield of sunflower was also reported by Khan and Akmal (2016).

Table 12: Harvest index (\%) of different varieties of sunflower as influenced by seed priming and planting. geometry.

\begin{tabular}{|c|c|c|c|c|c|c|}
\hline \multirow{2}{*}{\multicolumn{2}{|c|}{$\begin{array}{l}\text { Varie- Seed prim- } \\
\text { ties ing sources }\end{array}$}} & \multicolumn{4}{|c|}{ Planting geometry $(\mathrm{cm})$} & \multirow[t]{2}{*}{ Mean } \\
\hline & & $55 \times 35$ & $65 \times 30$ & $75 \times 25$ & $85 \times 20$ & \\
\hline \multirow[t]{5}{*}{$\mathrm{HO}-1$} & No priming & 20.6 & 23.7 & 21.6 & 25.4 & 22.8 \\
\hline & Canal water & 19.7 & 24.4 & 25.1 & 27.7 & 24.2 \\
\hline & $1.0 \%$ Urea & 25.8 & 25.4 & 26 & 29.7 & 26.7 \\
\hline & $0.2 \% \mathrm{ZnSO}_{4}$ & 27 & 27 & 28.4 & 29.9 & 28.1 \\
\hline & Mean & $23.3 c$ & $25.1 \mathrm{c}$ & $25.3 \mathrm{~b}$ & $28.2 \mathrm{a}$ & $25.5 \mathrm{a}$ \\
\hline \multirow{5}{*}{$\begin{array}{l}\text { Hys- } \\
\text { un-33 }\end{array}$} & No priming & 19.8 & 20.6 & 24.5 & 24.9 & 22.5 \\
\hline & Canal water & 20.3 & 23.6 & 24.9 & 28.6 & 24.4 \\
\hline & $1.0 \%$ Urea & 25.3 & 24.3 & 25.8 & 29.4 & 26.2 \\
\hline & $0.2 \% \mathrm{ZnSO}_{4}$ & 27.8 & 26.6 & 27.7 & 29.8 & 28.0 \\
\hline & Mean & $23.3 \mathrm{c}$ & $23.8 \mathrm{~b}$ & $25.7 \mathrm{~b}$ & $28.2 \mathrm{a}$ & $25.2 \mathrm{~b}$ \\
\hline \multirow{5}{*}{$\begin{array}{l}\text { Aver- } \\
\text { ages }\end{array}$} & No priming & $20.2 \mathrm{gh}$ & $22.2 \mathrm{fg}$ & $23.1 \mathrm{fg}$ & $25.2 \mathrm{e}$ & $22.6 \mathrm{~d}$ \\
\hline & Canal water & $20.0 \mathrm{~h}$ & $24.0 \mathrm{ef}$ & $25.0 \mathrm{de}$ & $28.2 \mathrm{ab}$ & $24.3 \mathrm{c}$ \\
\hline & $1.0 \%$ Urea & $25.6 \mathrm{de}$ & $24.9 \mathrm{de}$ & $25.9 \mathrm{cde}$ & $29.6 \mathrm{ab}$ & $26.5 \mathrm{~b}$ \\
\hline & $0.2 \% \mathrm{ZnSO}_{4}$ & $27.4 \mathrm{abc}$ & $26.8 \mathrm{bcd}$ & $28.1 \mathrm{abc}$ & $29.9 \mathrm{a}$ & $28.0 \mathrm{a}$ \\
\hline & Mean & $23.3 \mathrm{c}$ & $24.5 \mathrm{~b}$ & $25.5 \mathrm{~b}$ & $28.2 \mathrm{a}$ & - \\
\hline \multicolumn{2}{|c|}{ Variables } & $\mathrm{SE}$ & & $\mathrm{p}$-value & & LSD 5 \\
\hline \multicolumn{2}{|c|}{ Priming $(\mathrm{P})$} & 0.4732 & & 0.0000 & & 0.9459 \\
\hline \multicolumn{2}{|c|}{ Geometry (G) } & 0.4732 & & 0.0000 & & 0.9459 \\
\hline \multicolumn{2}{|c|}{ Varieties $(V)$} & 0.3346 & & 0.0405 & & 0.6688 \\
\hline \multicolumn{2}{|l|}{$P \times G$} & 0.9464 & & 0.0001 & & 1.8918 \\
\hline \multicolumn{2}{|l|}{$\mathrm{P} \times \mathrm{V}$} & 0.6692 & & 0.4193 & & 1.3377 \\
\hline \multicolumn{2}{|l|}{$\mathrm{G} \times \mathrm{V}$} & 0.6692 & & 0.0000 & & 1.3377 \\
\hline \multicolumn{2}{|c|}{$\mathrm{P} \times \mathrm{G} \times \mathrm{V}$} & 1.3384 & & 0.0009 & & 2.6754 \\
\hline
\end{tabular}

June 2020 | Volume 36 | Issue 2 | Page 455 
Harvest index (\%)

Harvest index was significantly $(p \leq 0.05)$ impacted by seed priming, geometry, varieties, and interaction of seed priming $\mathrm{x}$ varieties, geometry $\mathrm{x}$ varieties whereas, non-substantially $(p>0.05)$ by seed priming $\mathrm{x}$ geometry, and seed priming $\mathrm{x}$ geometry $\mathrm{x}$ varieties (Table 12). The data revealed that seed priming with $0.2 \% \mathrm{ZnSO}_{4}$ resulted in more harvest index, followed by seed priming with $1.0 \%$ Urea. The geometry of 85 x $20 \mathrm{~cm}$ gave highest harvest index, followed by 75 x $25 \mathrm{~cm}$. The HO-1 proved better in performance over Hysun-33. The interaction of HO-1 x seed priming with $0.2 \% \mathrm{ZnSO}_{4}$ gave higher harvest index. The interaction of HO-1 x geometry of $85 \times 20 \mathrm{~cm}$ resulted in greater harvest index. A boost in harvest index of sunflower was observed by seed priming (Jafri et al., 2015). Planting densities affected positively to sunflower harvest index (Ion et al., 2015).

Table 13: Oil content (\%) of different varieties of sunflower as influenced by seed priming and planting geometry.

\begin{tabular}{|c|c|c|c|c|c|c|}
\hline \multirow{2}{*}{$\begin{array}{l}\text { Varie- } \\
\text { ties }\end{array}$} & \multirow{2}{*}{$\begin{array}{l}\text { Seed prim- } \\
\text { ing sources }\end{array}$} & \multicolumn{4}{|c|}{ Planting geometry $(\mathrm{cm})$} & \multirow[t]{2}{*}{ Mean } \\
\hline & & $55 \times 35$ & $65 \times 30$ & $75 \times 25$ & $85 \times 20$ & \\
\hline \multirow[t]{5}{*}{$\mathrm{HO}-1$} & No priming & 36.7 & 36.9 & 37.1 & 37.5 & 37.1 \\
\hline & Canal water & 38.1 & 39.0 & 39.7 & 39.7 & 39.1 \\
\hline & $1.0 \%$ Urea & 39.7 & 40.0 & 41.0 & 41.7 & 40.6 \\
\hline & $0.2 \% \mathrm{ZnSO}_{4}$ & 40.4 & 41.8 & 42.4 & 42.7 & 41.8 \\
\hline & Mean & 38.7 & 39.4 & 40.1 & 40.4 & $39.7 \mathrm{~A}$ \\
\hline \multirow{5}{*}{$\begin{array}{l}\text { Hys- } \\
\text { un-33 }\end{array}$} & No priming & 35.8 & 36.0 & 36.3 & 36.7 & 36.2 \\
\hline & Canal water & 37.6 & 38.4 & 38.5 & 38.6 & 38.3 \\
\hline & $1.0 \%$ Urea & 38.5 & 39.3 & 39.4 & 40.6 & 39.5 \\
\hline & $0.2 \% \mathrm{ZnSO}_{4}$ & 39.0 & 40.9 & 41.2 & 41.4 & 40.6 \\
\hline & Mean & 37.7 & 38.7 & 38.9 & 39.3 & $38.6 \mathrm{~B}$ \\
\hline \multirow{5}{*}{$\begin{array}{l}\text { Aver- } \\
\text { ages }\end{array}$} & No priming & $36.3 \mathrm{~h}$ & $36.5 \mathrm{gh}$ & $36.7 \mathrm{gh}$ & $37.1 \mathrm{~g}$ & $36.6 \mathrm{~d}$ \\
\hline & Canal water & $37.9 \mathrm{f}$ & 38.7 e & 39.1de & $39.2 \mathrm{de}$ & $38.7 \mathrm{c}$ \\
\hline & $1.0 \%$ Urea & $39.1 \mathrm{de}$ & $39.7 \mathrm{~cd}$ & $40.2 \mathrm{c}$ & $41.2 \mathrm{~b}$ & $40.0 \mathrm{~b}$ \\
\hline & $0.2 \% \mathrm{ZnSO}_{4}$ & $39.7 \mathrm{~cd}$ & $41.4 \mathrm{~b}$ & $41.8 \mathrm{ab}$ & $42.1 \mathrm{a}$ & $41.2 \mathrm{a}$ \\
\hline & Mean & $38.2 \mathrm{~d}$ & $39.0 \mathrm{c}$ & $39.5 \mathrm{~b}$ & $39.9 \mathrm{a}$ & - \\
\hline \multicolumn{2}{|c|}{ Variables } & SE & & $\mathrm{p}$-value & & LSD 5\% \\
\hline \multicolumn{2}{|c|}{ Priming $(\mathrm{P})$} & 0.1590 & & 0.0000 & & 0.3179 \\
\hline \multicolumn{2}{|c|}{ Geometry (G) } & 0.1590 & & 0.0000 & & 0.3179 \\
\hline \multicolumn{2}{|c|}{ Varieties (V) } & 0.1125 & & 0.0000 & & 0.2248 \\
\hline \multicolumn{2}{|l|}{$P \times G$} & 0.3181 & & 0.0061 & & 0.6358 \\
\hline \multicolumn{2}{|l|}{$\mathrm{P} \times \mathrm{V}$} & 0.2249 & & 0.4816 & & 0.4496 \\
\hline \multicolumn{2}{|l|}{$\mathrm{G} \times \mathrm{V}$} & 0.2249 & & 0.6564 & & 0.4496 \\
\hline \multicolumn{2}{|c|}{$\mathrm{P} \times \mathrm{G} \times \mathrm{V}$} & 0.4498 & & 0.9731 & & 0.8992 \\
\hline
\end{tabular}

Oil content (\%)

The value of sunflower crop depends upon its oil content percentage. The analysis of variance illustrated a substantial $(p \leq 0.05)$ impact on oil content for seed priming, geometry, varieties, and interaction of seed priming $\mathrm{x}$ geometry while non-substantial $(p>0.05)$ effect was noticed for interaction of seed priming $\mathrm{x}$ varieties, geometry $\mathrm{x}$ varieties, and seed priming $\mathrm{x}$ geometry $\mathrm{x}$ varieties (Table 13). The results showed that highest oil content was noted in seed priming with $0.2 \% \mathrm{ZnSO}_{4}$, followed by seed priming with $1.0 \%$ Urea. The planting geometry of $85 \times 20 \mathrm{~cm}$ gave greatest oil content, followed by $75 \times 25 \mathrm{~cm}$. The variety HO-1 resulted in higher oil content than Hysun-33. The interaction of seed priming with $0.2 \%$ $\mathrm{ZnSO}_{4} \times$ geometry of $85 \times 20 \mathrm{~cm}$ gave highest oil content. The higher oil content with zinc priming may be attributed to active role of zinc for several enzymes (Rehman et al., 2015). Shinde and Gautam (2016) suggested significant effect of planting densities on sunflower oil content.

\section{Conclusions and Recommendations}

The results suggested that seed primed with $0.2 \%$ $\mathrm{ZnSO}_{4}$ and planted as $85 \times 20 \mathrm{~cm}$ conferred higher growth, better yield components that resulted higher yield and oil content of sunflower. The variety HO-1 is best option to yield as compared to another available hybrid Hysun-33.

\section{Acknowledgments}

This is original research article prepared from $\mathrm{PhD}$ dissertation of main author submitted at Sindh Agriculture University Tandojam.

\section{Novelty Statement}

The present research work investigated the effect of seed priming with $\mathrm{ZnSO}_{4}$ and planting geometry on growth and yield of sunflower, which could help in obtaining quality seed in the local conditions.

\section{Author's Contribution}

ZAA planned experiment, gathered data and prepared manuscript. MNK guided scholar as a whole from designing experiment to writing of manuscript. AAS provided research facility and helped in manuscript write-up. NL contributed in research material and data analysis. MIK helped in interpretation of results and editing of manuscript. ANS contributed in collection 
of data and proof reading of manuscript. MBA helped in tabulation and correction of manuscript.

\section{Conflict of interest}

There is no conflict of interest among authors.

\section{References}

Akmal, M., M. Asim and M. Gilbert. 2014. Influence of seasonal variation on radiation use efficiency and crop growth of maize planted at various densities and N-rates. Pak. J. Agric. Sci., 51: 835-846.

Amjad, M., 2014. Oil seeds crops of Pakistan. Pak. Agric. Res. Council Islamabad, pp. 1-40.

Amjed, R., R. Ali and I.R. Noorka. 2013. Differential growth and development response of sunflower hybrid in contrasting irrigation regimes. Am. J. Sci., 13(4): 157-163.

Bakht, J., M. Shafi, M. Yousaf, R. Din and M.A. Khan. 2010. Effect of irrigation on physiology and yield of sunflower hybrids. Pak. J. Bot., 42(2): 1317-1326.

Buriro, M., A.S. Sanjrani, Q.I. Chachar, N.A. Chachar, S.D. Chachar, B. Buriro, A.W. Gandahi and T. Mangan. 2015. Effect of water stress on growth and yield of sunflower. Int. J. Agric. Technol., 2(1): 260- 270.

Chatterjee, N., D. Sarkar, A. Sankar, S. Pal, H.B. Singh, R.K. Singh and J.S. Bohra. 2018. Onfarm seed priming interventions in agronomic crops. Acta Agric. Slov., 111(3): 1-12. https:// doi.org/10.14720/aas.2018.111.3.19

Dawa1, S., F.U. Khan, Neelofar, F.A. Khan, P. Tundup and T. Angdu. 2017. Effect of different priming methods on growth and flowering of different flowers of Kashmir Valley India. Int. J. Curr. Microbiol. App. Sci., 6(12): 3333-3344. https://doi.org/10.20546/ijcmas.2017.612.388

Farooq, M., A. Wahid and K.H.M. Siddique. 2012. Micronutrient application through seed treatments. A review. Soil Sci. Plant Nutr., 12: 125-142. https://doi.org/10.4067/S071895162012000100011

GoP, 2018. Economic survey of Pakistan 2017-18, Government of Pakistan, ministry of food, agriculture and livestock division, economic affairs wing, Islamabad, Pakistan.

Halmer, P., 2003. Methods to improve seed performance. In: Benech-Arnold, R.L., Sanchez, R.A. (eds) Seed Physiology, Applications to Agricul- ture. Food Product Press, New York, pp. 40-48.

Hekmat, N.M., 2013. Effect of planting dates and plant spacing on growth yield and yield components of sunflower (Helianthus annuus L.) in Iraqi Kurdistan region. JZS-A, 15(4): 1-4. https://doi.org/10.17656/jzs.10275

Ion, V., G. Dicu, M. Basa, M. Dumbrava, G. Temocico, L.L. Eupure and D. State. 2015. Sunflower yield and yield components under different sowing conditions. Agric. Agric. Sci. Proc., (6): 44-51. https://doi.org/10.1016/j.aaspro.2015.08.036

Jafri, N., M. Mazid and F. Mohammad. 2015. Responses of seed priming with gibberellic acid on yield and oil quality of sunflower (Helianthus annuus L.) Indian J. Agric. Res., 49(3): 235-240. https://doi.org/10.5958/0976058X.2015.00036.0

Kalaiyarasan, C. and V. Vaiyapuri. 2007. Effect of integrated nutrient management practices on growth and yield attributes and quality characters of sunflower (Helianthus annuus L.). Int. J. Trop. Agric., (35): 15-18.

Kazemeini, S.A., M. Edalat and A. Shekoofa. 2009. Interaction effects of deficit irrigation and row spacing on sunflower (Helianthus annuus L.) growth, seed yield and oil yield. Afr. J. Agric. Res., 4(11): 1165-1170.

Khalil, I.A.K. and A. Jan. 2010. Cropping technology, Text book of agriculture. Third edition, National Book Foundation Islamabad., pp. 247248.

Khan, M.A., and M. Akmal. 2016. Plant arrangement effect on the sunflower yield and yield traits in spring season crop. Sarhad J. Agric., 32(3): 151-155. https://doi.org/10.17582/journal.sja/2016.32.3.151.155

Kouchebagh, S.B., F. Farahvah, B. Mirshekari, H.A. Kazemi and F.R. Khoei. 2014. Seed priming techniques may improve grain and oil yields of sunflower (Helianthus annuus L.). J. Anim. Plant Sci., 24(6): 1863-1868.

Mahmood, A., M.F. Saleem, M. Tahir, M.A. Sarwar, T. Abbas, A. Zohaib and H.T. Abbas. 2018. Sunflower (Helianthus annuus L.) growth, yield and oil quality response to combined application of nitrogen and boron. Pak. J. Agric. Res., 31(1): 86-97. https://doi.org/10.17582/journal. pjar/2018/31.1.86.97

Mehrpouyan, M., A. Nazari-Golshan and S. Sayfzadea. 2010. Effect of irrigation stop at differ- 
ent growth stages on some agronomic traits of sunflower (Helianthus annuus) under three plant densities in Takestan region, Iran. Plant Ecophysiol., (2): 137-144.

Moeinzadeh, A., F.S. Zadehl, M.A. zadeh and F.H. Tajabadi. 2010. Biopriming of sunflower (Helianthus annuus L.) seed with Pseudomonas fluorescens for improvement of seed invigoration and seedling growth. Agric. J. Crop Sci., 4(7): 564-570.

Mokhtari, A., and S.G. Moosavi. 2016. Effect of plant density on some morphological traits, yield and yield components of sunflower $(\mathrm{He}-$ lianthus annuus L.) cultivars. JPEC., 10(34): 132-144.

Mrda,J., J. Crnobarac, N. Dusanic, V. Radic, D. Miladinovic, S. Jocic and V. Miklic. 2010. Effect of storage period and chemical treatment on sunflower seed germination. HELIA., 33(53): 199206. https://doi.org/10.2298/HEL1053199M

Rashid, A., and K.S. Memon. 2001. Soil science. National Book Foundation Islambabad, pp. 372-379.

Rasool, K., A. Wajid, Sanaullah, A. Ghaffar, M. Shoaib, M. Arshad and S. Abbas. 2015. Optimizing nitrogen rate and planting density for sunflower under irrigated conditions of Punjab. SAARC. J. Agric., 13(1): 174-187. https://doi. org/10.3329/sja.v13i1.24190

Rayan, J., G. Estefan and A. Rashid. 2001. Soil and plant analysis laboratory manual. Int. Center Agric. Res. the Dry Areas (ICARDA), Aleppo, Syria.pp. 172.

Rehman, A., M. Farooq, R. Ahmad and S.M.A. Basra. 2015. Seed priming with zinc improves the germination and early seedling growth of wheat. Seed Sci. Tech., 43(2): 262-268. https:// doi.org/10.15258/sst.2015.43.2.15

Shah, N., K. Aujla, M. Ishaq and A. Farooq. 2013. Trends in sunflower production and its poten- tial in increasing domestic edible oil production in Punjab, Pakistan. Sarhad J. Agric., (29): 7-13. Shinde, L.D. and G. Gautam. 2016. Effect of plant geometry and sulphur levels on growth, yield and oil content of sunflower. J. Life Sci., 12(4): 973-981.

Siddiqui, M.H. 2010. Nutrient management for sunflower production $\mathrm{PhD}$ dissertation submitted to Sindh Agric. Univ. Tandojam, Pakistan.

Soomro, A.G., A.H. Memon, M.A. Gadehi, R.M. Memon, S.D. Junejo, S. Talpur and M.A. Memon. 2015. Growth and yield of sunflower in response to planting geometry and nitrogen foliar application at various crop stages. Ameri. Eura. J. Agric. Environ. Sci., 15 (1): 140-146.

Statistix, 2006. Statistix 8.1 user guide, version 1.0. Analytical Software, PO Box 12185, Tallahassee FL 32317 USA. Copy right 2006 by Analytical Software.

Tavakoli, A. 2013. Effect of plant density on yield and yield components of sunflower varieties in temperate regions of Kermanshah. Eur. J. Exp. Biol., 3(5): 601-604.

Tengong, S., P. Sinkeng, M.H, Linglee and Y. Tsehung. 2010. Equilibrium studies for the removal of dye by sunflower seed husk. Int. J. Phys. Sci., 5(8): 1270-1276.

Vijayaakshmi, K., N.K. Sanghi, W.L. Pelton and C.H. Anderson. 2017. Effects of plant population and row spacing on sunflower agronomy. Can. J. Plant Sci., (55): 491-499. https://doi. org/10.4141/cjps75-075

Zahoor, A., M. Riaz, S. Ahmad, H. Ali, M.B. Khan, K. Javed, M.A. Anjum, M.Z. Haq and M. Khan. 2010. Ontogeny growth and radiation use efficiency of (Helianthus annuus L.) as affected by hybrids, nitrogenous regimes and planting geometry under irrigated arid conditions. Pak. J. Bot., 42(5): 3197-3207. 